\title{
Tendances évolutives de la rougeole en Commune VI du District de Bamako de 2014 à 2018
}

\section{Trends in measles in Commune Vl of the District of Bamako from 2014 to 2018}

Ouattara $\mathrm{S}^{1^{*}}$, Sangho $\mathrm{O}^{2,3}$, Yalcouyé $\mathrm{H}^{4}$, Traoré $\mathrm{B}^{5}$, Kayembé Ken ${ }^{5}$, Coulibaly $\mathrm{CA}^{3}$, Telly $\mathrm{N}^{3}$, Tounkara $\mathrm{M}^{3}$, Diarra $\mathrm{B}^{6}$, Konaké $\mathrm{MS}^{6}$, Kéita $\mathrm{H}^{7}$, Coulibaly $\mathrm{S}^{8}$ Ballayira $\mathrm{Y}^{9}$, Koné $\mathrm{Y}^{7}$, Diakité $\mathrm{S}^{10}$, Togola $\mathrm{OB}^{11}$, Ag Intagrist $\mathrm{M}^{13}$, Coulibaly $\mathrm{H}^{12}$, Barry $\mathrm{D}^{8}$

1. Direction Régionale de la Santé de Bamako, Mali

2. DER des Sciences Biologiques et Médicales, FAPH/USTTB, Mali

3. Département d'Enseignement et de Recherche en Santé Publique, FMOS/USTTB, Mali

4. Direction Nationale des Services Vétérinaires, Bamako, Mali

5. Réseau Africain des Epidémiologiste de Terrain, AFENETMali, DGSHP, Bamako, Mali

6. Centre de Santé de Référence de la Commune VI, Bamako, Mali

7. Direction Générale de la Santé et de l'Hygiène Publique (DGSHP), Bamako, Mali

8. Institut National de Santé Publique, INSP, Bamako, Mali

9. Cellule Sectorielle de Lutte Contre le SIDA, la Tuberculose et les Hépatites Virales (CSLS-TBH), Bamako, Mali

10. Direction Régionale de la Santé de Sikasso, Mali

11. Centre de Santé de Référence de Tominian, Mali

12. Direction Régionale de la Santé de Koulikoro, Mali

13. Centre de Santé de Référence de Niono, Mali

*Auteur correspondant : Dr Seydou Ouattara, Direction Régionale de la Santé de Bamako, Mali, 782640 46, drouattaraseydou@gmail.com

\section{Résume :}

Introduction : La rougeole est une maladie éruptive fébrile, due à un virus de la famille des Paramyxoviridae (Morbillivirus) qui se transmet par voie aérienne, d'une personne à l'autre, par les gouttelettes respiratoires en suspension dans l'air. Le Mali a enregistré 87 cas de rougeole 2016, 17 cas en 2017 et 414 cas en 2018. L'objectif était de décrire les cas suspects et confirmés en personnes, temps, lieu et déterminer les tendances évolutives de la rougeole en Commune IV de 2014 à 2018. Matériel et méthodes : il s'agissait d'une étude transversale descriptive en déroulée en Juin 2019. La population d'étude était constituée des cas suspects et confirmés de rougeole enregistrés dans la base de données de surveillance. Nos données ont été saisies et analysées sur Excel 2013 et Epi Info 7. Résultats : L'enquête a porté sur un total de 213 cas dont 39 positifs soit une fréquence de $18,31 \%$. La moyenne d'âge était de 4,98 ans et 5,50 ans respectivement chez les positifs. Les enfants de moins 5 ans ont été les plus atteints autant pour la suspicion que pour la confirmation. C'est à Sénou (28 cas) et à Yirimadio (34 cas) que venaient les plus grands nombres de cas notifiés. De 2014 à 2018, il y a eu deux épidémies notamment en 2015 et 2018. Tous les cas ont été guéris sauf trois qui sont décédés en 2018 à Sokorodji. Conclusion : Les enfants de moins 5 ans étaient plus atteints. Deux épidémies sont survenues. Tous les cas ont été guéris sauf 3 décédés. La compréhension de la dynamique de cette maladie nécessitera l'évaluation des facteurs déterminants de la survenue de la rougeole en commune VI.

Mots clés : rougeole, tendance, épidémie, Bamako, Mali

\begin{abstract}
:
Introduction: Measles is a febrile rash disease caused by a virus of the Paramyxoviridae family (Morbillivirus) that is transmitted by air, from person to person, through respiratory droplets suspended in the air. Mali recorded 87 cases of measles in 2016, 17 cases in 2017 and 414 cases in 2018. The objective was to describe the suspected and confirmed cases in person and time and to determine the evolving trends of measles in commune IV from 2014 to 2018. Material and methods: this was a descriptive cross-sectional study carried out in June 2019. The study population consisted of suspected and confirmed cases of measles recorded in the surveillance database. Our data were entered and analyzed in Excel 2013 and Epi Info 7. Results: The investigation covered a total of 213 cases, 39 of which were positive, ie a frequency of $18.31 \%$. The mean age was 4.98 years and 5.50 years respectively for the positive. Children under 5 years of age were the most affected both for suspicion and confirmation. The largest numbers of reported cases came from Sénou (28cas) and Yirimadio (34cas). From 2014 to 2018 there were two epidemics, notably in 2015 and 2018. All the cases were cured except three who died in 2018 in Sokorodji. Conclusion: Children under 5 years old were more affected. Two epidemics occurred. All cases were cured except 3, who died. Understanding the dynamics of this disease will require evaluating the determining factors for the occurrence of measles in Commune VI.
\end{abstract}

Keywords: measles, trend, epidemic, Bamako, Mali

\section{Introduction}

La rougeole est une maladie éruptive fébrile, due à un virus de la famille des Paramyxoviridae (Morbillivirus) qui se transmet par voie aérienne, d'une personne à l'autre, par les gouttelettes respiratoires en suspension dans l'air (1). Dans de nombreux pays africains, la rougeole est la quatrième cause majeure de mortalité chez les enfants de moins de 5 ans (1).

Depuis 2017, après une tendance à la baisse (diminution de 586701 cas en 2005 à 132328 cas en 2016, soit une baisse de $75 \%$ ), on observe une augmentation des cas qui perdure en 2019 notamment dans les régions Europe et Amériques (2). En 2018, le total des cas déclarés à I'OMS dans le monde est supérieur à 300000 cas (2). Des flambées épidémiques ont été observées, notamment dans la région des Amériques (Brésil 10326 cas, Vénézuela 5643 cas, États-Unis 372 cas) et en Europe (Ukraine 53218 cas, France 2953 cas, Italie 2953 cas) (2). Le nombre de cas déclarés pour 2019 à la fin du mois de mars est égal à 112163 soit une augmentation de 25 
\% par rapport à la même période de 2018 (2). Les deux régions OMS les plus touchées sont la région Afrique avec 59943 cas (augmentation de 386 \%) et la région Europe avec 34383 cas (augmentation de 72 \%) (2). En revanche, I'OMS trouve que la rougeole peut et doit être éradiquée (3). D'où la nécessité de stratégies novatrices avec l'opportunité que la vaccination anti rougeoleux est un bon indicateur de performance des systèmes de vaccination et de santé (4). Certains pays comme le Kenya ont fait des progrès dans le sens de l'élimination même s'il reste discutable avec les couvertures de deuxième dose faible (5). D'où l'intérêt des sciences sociales pour combattre les réticences à la vaccination (6).

Selon les rapports annuels de la surveillance épidémiologique de la DGSHP, le Mali a enregistré 87 cas de rougeole en 2016, 17 cas en 2017 et 414 cas en 2018 (7-9).

La surveillance est une approche attentive, méthodique, de collecte, de compilation, d'analyse, de diffusion de l'information dans un but d'évaluation et de prise de décision à temps utile qui sert à améliorer ou à maintenir la santé de la population (1).

Le système de surveillance de la rougeole comporte la détection des cas suspects au niveau des centres de santé, la notification à l'échelon supérieur, le prélèvement et l'envoie des échantillons au laboratoire national de référence. La surveillance cas par cas de la rougeole est intégrée à la « Surveillance Intégrée de la Maladie et la Riposte » (SIMR) (10). Les échantillons prélevés par les formations sanitaires sont envoyés au Laboratoire national de référence située à l'Institut National de Recherche en Santé Publique (INRSP) (11). Après analyse, les résultats (rétro information) sont envoyés à la Division Prévention et Lutte contre la Maladie (DPLM), à l'Organisation Mondiale de la Santé (OMS) et la formation sanitaire où l'échantillon a été prélevé (11).

Notre objectif était de décrire les cas suspects et confirmés en personnes temps lieu et déterminer les tendances évolutives de la rougeole en commune IV de 2014 à 2018. Notre étude avait pour but de renforcer le système de surveillance de la rougeole dans cette commune de Bamako.

\footnotetext{
Matériel et méthodes

Nous avons réalisé une étude transversale descriptive en Commune VI du district de Bamako en Juin 2019. La population d'étude était constituée de tous les cas suspects et confirmés de rougeole enregistrés en commune VI du 1er janvier 2014 au 31 décembre 2018. Nous avons aussi inclus tous résultats des examens effectués dans le cadre de la surveillance des cas par cas de la rougeole du 1er Janvier 2014 au 31 Décembre 2018. Nous n'avons pas inclus les données incomplètes. Nous avons collecté les données à partir de la base de données sur la rougeole. Nos données ont été saisies et analysées sur les logiciels Microsoft Excel 2013 et Epi Info 7. Nous avons effectué des comparaisons de moyennes avec le test $t$ de Student, et des celles des proportions avec le Khi
}

Deux de Pearson ou le test exact de Fisher selon leur condition d'application.

Considérations éthiques et déontologiques :

Nous avons demandé et obtenu l'autorisation des autorités sanitaires avant le début de notre étude. Les noms et prénoms n'ont pas été collectés. Les données ne permettent pas de remonter jusqu'aux individus. La confidentialité et l'anonymat ont été garantis.

\section{Résultats}

L'enquête a porté sur un total de 213 cas dont 39 positifs soit une fréquence de $18,31 \%$. Les caractéristiques sociodémographiques des enquêtés sont présentées dans le tableau I. La moyenne d'âge chez les positifs était 4,98 ans $\pm 6,93$ ans. La moyenne d'âge chez les négatifs est 5,50 ans $\pm 4,80$ ans. La majorité des cas était les enfants. C'est à Sénou (28 cas) et à Yirimadio (34 cas) que venaient les plus grands nombres de cas notifiés (tableau I).

La majorité des cas a été notifiée par le CSRéf de la commune 6 soit $51 \%$ cas suivi de ASACOYIR avec $9 \%$ cas, ASACOSE et ASACOBAFA avec $8 \%$ cas. Sénou a enregistré le plus grand nombre de cas positifs (tableau l).

La durée moyenne entre le début et la notification de la maladie était de $2.62 \pm 1.63$ jours chez les cas positifs et $2.93 \pm 3.57$ chez les négatifs (tableau II).

C'est en 2015 et 2018 que la commune a enregistré des cas positifs, 26 positifs soit $66,67 \%$ en 2015 et 13 cas soit 18,06\% en 2018 (figure 1).

La plupart des cas notifiés étaient vaccinés soit $62.26 \%$ et $26 \%$ n'étaient pas vaccinés, le statut vaccinal pour $11.79 \%$ n'était pas disponible.

Tous les cas notifiés de 2014 à 2017 sont guéris, cependant c'est en 2018 que la commune a enregistré 3 décès (figure 2).

\section{Discussion}

Moins d'un cinquième de cas suspects ont été confirmés au laboratoire. Cependant, à Addis Abeba en Ethiopie, près du tiers $(27,5 \%)$ a été confirmé dans leur analyse des données de 2005 à 2014 (12). L'incidence était de 37\% durant l'épidémie de 2018-2019 à Madagascar (13). Beaucoup de pays œuvrent dans le cadre de l'élimination de la rougeole. Cependant malgré la réussite de cette élimination, avec à l'appui la certification par l'OMS dont la Mongolie, les cas continuent de surgir (14).

La moyenne d'âge chez les positifs est 4,98 ans ce qui voudrait dire que les enfants de moins de 5 ans étaient les plus susceptibles. Cette tendance a été notée au Nigéria, lors de l'analyse des données de 5 ans de 2014 à 2018, avec $64,1 \%$ des cas entre 1-4ans et l'âge était un prédicteur de la rougeole (15) et au Liban (16). Le sexe ne semble pas avoir une influence sur la survenue de la maladie car leur relation n'était significative. Cela est comparable aux résultats du Nigéria où $54,6 \%$ était masculin (15). 
C'est à Sénou (28 cas) et à Yirimadio (34 cas) que le plus grand nombre de cas ont été notifiés. Cela pourrait s'expliquer par l'étendue de leurs superficies, la pauvreté relative et la proximité. La majorité des cas a été notifiée par le CSRéf de la commune 6 soit $51 \%$. Cela peut s'expliquer par le fait que le CSRéf fait la majorité des consultations. C'est Sénou qui a enregistré le plus grand nombre de cas positifs ce qui met en cause la couverture vaccinale dans cette aire de santé.

La durée moyenne entre le début et la notification de la maladie est de 2,62 jours chez les cas positifs et $2.93 \mathrm{chez}$ les négatifs. C'est en 2015 et 2018 que la commune a enregistré des cas positifs, 26 positifs soit $66,67 \%$ en 2015 et 13 cas soit $18,06 \%$ en 2018 . Ce qui montre une diminution de l'incidence. Cependant, il est à noter que la plupart des cas notifiés étaient vaccinés soit $62.26 \%$. Cette fréquence montre que $37,74 \%$ n'étaient pas vaccinés. Ce qui interpelle les acteurs du système de santé en termes de qualité de vaccin et de la vaccination. Ce résultat est inférieur à celui de (Tangara) qui avait trouvée $91,74 \%$ de couverture vaccinale (17). Une enquête de couverture vaccinale réalisée en 2010 a permis d'enregistrer $62 \%$ de couverture vaccinale en Vaccin Anti Rougeoleux (VAR) chez les enfants de 12 à 23 mois sur un objectif national de 95\% (10), comparable au résultat de notre étude. Cependant, l'Organisation Mondiale de la Santé signale une augmentation de la première dose de vaccination contre la rougeole synonyme de réduction de l'incidence de son incidence (3). Certains auteurs pensent même plus loin en proposant de vacciner les parents pour prétendre à une élimination de la rougeole, comme par exemple en Italie où plus la proportion de vaccinée estimée est élevée, plus sera tôt l'élimination (18).

Le statut vaccinal pour $11.79 \%$ n'était pas disponible. Cela pourrait s'expliquer par le fait que les agents ne demandent pas systématiquement le statut vaccinal pendant la consultation ou l'accompagnant n'avait pas l'information. L'absence de vaccination ou la méconnaissance du statut vaccinal sont des facteurs de risque de la rougeole. Au Liban, $52,26 \%$ des cas étaient notés chez les non vaccinés contre $9,66 \%$ chez les vaccinées d'avec 2 doses et plus (16).

En fonction de l'évolution de la maladie, tous les cas notifiés de 2014 à 2017 sont guéris, cependant c'est en 2018 que la commune a enregistré 3 décès. Ces décès ont tous été notifiés dans la même zone à Socorodji. L'étude de Lee CT et al. note une proportion de décès de $0,13 \%$ et $2,31 \%$ dans ses deux vagues d'épidémie en 2019 (19). Cette situation de létalité de la maladie est d'autant plus préoccupante que des auteurs ont étudié la physiopathologie en trouvant l'immunosuppression qu'elle provoque et la sévérité qui dépend non seulement de l'âge du patient mais aussi du contexte sanitaire dans lequel l'épidémie survient (20).

Par ailleurs $81.60 \%$ des cas notifiés étaient négatifs. Cela pose un problème de diagnostic différentiel de la rougeole et d'insuffisance du niveau de compétence des prestataires de santé dans la commune. En Ethiopie, la compatibilité du diagnostic clinique avec le laboratoire était de $12,2 \%$ (12). Cette étude pose le problème de qualité des données sur la couverture vaccinale dans la commune. En effet, la plupart des informations sur le statut vaccinal étaient verbales faute de retrouver la carte. Notre étude ne permettait pas d'analyser les facteurs déterminants de la rougeole, importants pour déterminer les raisons de la survenue de ces épidémies de façon presque cyclique.

Limites de l'étude : certaines données non disponibles ou difficiles d'accès faute de bon archivage.

\section{Conclusion}

Les enfants de moins 5 ans ont été les plus atteints autant pour la suspicion que pour la confirmation. Les aires de santé de Sénou et Yirimadio ont enregistrées le maximum de cas. De 2014 à 2018, il y a eu deux épidémies notamment en 2015 et 2018.Tous les cas ont été guéris sauf trois décédés en 2018 à Sokorodji. Pour mieux comprendre la dynamique de cette maladie, il serait intéressant d'évaluer les facteurs déterminants de la survenue de la rougeole en commune VI. D'ici là, la formation des prestataires de santé sur les maladies évitables par la vaccination serait une bonne alternative.

\section{Références}

1. MINISTERE DE LA SANTE, D. Guide

Technique Rougeole. Section Surveillance

Epidémiologique; 2017.

2. MIGLIANI R. L'épidémiologie de la rougeole dans le monde en avril 2019 [Internet]. L'épidémiologie de la rougeole dans le monde en avril 2019. 2019 [cité 15 janv 2021]. Disponible sur:

http://www.mesvaccins.net/web/news/13735-|epidemiologie-de-la-rougeole-dans-le-monde-en-avril2019

3. Coughlin MM, Beck AS, Bankamp B, Rota PA. Perspective on Global Measles Epidemiology and Control and the Role of Novel Vaccination Strategies. Viruses [Internet]. 19 janv 2017 [cité 15 janv 2021];9(1). Disponible sur: https://www.ncbi.nlm.nih.gov/pmc/articles/PMC5294980/ 4. Goodson JL. Recent setbacks in measles elimination: the importance of investing in innovations for immunizations. Pan Afr Med J [Internet]. 20 févr 2020 [cité 15 janv 2021];35(Suppl 1). Disponible sur: https://www.ncbi.nlm.nih.gov/pmc/articles/PMC7196335/ 5. Kisangau N, Sergon K, Ibrahim Y, Yonga F Langat $D$, Nzunza $R$, et al. Progress towards elimination of measles in Kenya, 2003-2016. Pan Afr Med J [Internet]. 28 sept 2018 [cité 15 janv 2021];31. Disponible sur:

https://www.ncbi.nlm.nih.gov/pmc/articles/PMC6457729/ 6. Kornbluh R, Davis R. Global trends in measles publications. Pan Afr Med J [Internet]. 20 févr 2020 [cité 15 janv 2021];35(Suppl 1). Disponible sur: https://www.ncbi.nlm.nih.gov/pmc/articles/PMC7195917/ 
7. Direction Nationale de la Santé. Rapport Annuel 2016. Bamako, Mali: Ministère de la Santé; 2017. 8. Direction Nationale de la Santé. Rapport Annuel 2017. Bamako, Mali: Ministère de la Santé; 2018. 9. Direction Nationale de la Santé. Rapport Annuel 2018. Bamako, Mali: Ministère de la Santé; 2019. 10. MINISTERE DE LA SANTE, Direction Nationale de la Santé. Plan stratégique national d'élimination de la rougeole au Mali, 2013-2020 [Internet]. DNS; 2012 [cité 15 janv 2021]. Disponible sur: http://staging.nationalplanningcycles.org/sites/default/files /planning_cycle_repository/mali/plan_strategique_elimina tion_rougeole_sikasso_mali_2013_2020_21-08013 vf $0 . p d f$

11. MINISTERE DE LA SANTE, D. Guide intégré de la surveillance des maladies, nouvelles version. Division Surveillance Epidémiologique; 2017.

12. Hassen MN, Woyessa AB, Getahun M, Beyene $B$, Buluanger $L$, Ademe A, et al. Epidemiology of measles in the metropolitan setting, Addis Ababa, Ethiopia, 20052014: a retrospective descriptive surveillance data analysis. BMC Infect Dis [Internet]. 14 août 2018 [cité 15 janv 2021];18. Disponible sur:

https://www.ncbi.nlm.nih.gov/pmc/articles/PMC6092798/

13. Nimpa MM, Andrianirinarison JC, Sodjinou VD, Douba A, Masembe YV, Randriatsarafara F, et al. Measles outbreak in 2018-2019, Madagascar: epidemiology and public health implications. Pan Afr Med J [Internet]. 19 mars 2020 [cité 15 janv 2021];35.

Disponible sur:

https://www.ncbi.nlm.nih.gov/pmc/articles/PMC7250232/ 14. Orsoo O, Saw YM, Sereenen E, Yadamsuren B, Byambaa A, Kariya T, et al. Epidemiological characteristics and trends of a Nationwide measles outbreak in Mongolia, 2015-2016. BMC Public Health [Internet]. 15 févr 2019 [cité 15 janv 2021];19. Disponible sur:

https://www.ncbi.nlm.nih.gov/pmc/articles/PMC6377723/ 15. Aworabhi-Oki N, Numbere T, Balogun MS, Usman A, Utulu R, Ebere N, et al. Trends in measles cases in Bayelsa state, Nigeria: a five-year review of case-based surveillance data (2014-2018). BMC Public
Health [Internet]. 15 juin 2020 [cité 15 janv 2021];20.

Disponible sur:

https://www.ncbi.nlm.nih.gov/pmc/articles/PMC7296655/

16. El Zarif T, Kassir MF, Bizri N, Kassir G,

Musharrafieh U, Bizri AR. Measles and mumps outbreaks in Lebanon: trends and links. BMC Infect Dis [Internet]. 26 mars 2020 [cité 15 janv 2021];20. Disponible sur: https://www.ncbi.nlm.nih.gov/pmc/articles/PMC7098136/ 17. Tangara $D$. Vaccination de routine des enfants et des mères d'enfants dans le village de kabalabougou, commune rurale du mande, cercle de Kati, région de Koulikoro [Internet]. [Bamako]: USTTB; 2014 [cité 15 janv 2021]. Disponible sur:

https://www.bibliosante.ml/handle/123456789/617

18. Marziano V, Poletti P, Trentini F, Melegaro A, Ajelli M, Merler S. Parental vaccination to reduce measles immunity gaps in Italy. eLife [Internet]. [cité 15 janv 2021];8. Disponible sur:

https://www.ncbi.nlm.nih.gov/pmc/articles/PMC6721460/ 19. Lee CT, Hagan JE, Jantsansengee B,

Tumurbaatar O-E, Altanchimeg S, Yadamsuren B, et al. Increase in Infant Measles Deaths During a Nationwide Measles Outbreak-Mongolia, 2015-2016. J Infect Dis. 22 oct 2019;220(11):1771-9.

20. Misin A, Antonello RM, Di Bella S, Campisciano G, Zanotta N, Giacobbe DR, et al. Measles: An Overview of a Re-Emerging Disease in Children and Immunocompromised Patients. Microorganisms [Internet]. 18 févr 2020 [cité 15 janv 2021];8(2). Disponible sur: https://www.ncbi.nlm.nih.gov/pmc/articles/PMC7074809/

\section{Remerciements}

Gouvernement malien (Ministère de la santé et des Affaires Sociales, Ministère de l'Elevage et de la Pêche, Ministère de l'Environnement) ; CDC/AFENET pour le financement de cette activité ; Formateurs et mentors pour leur accompagnement ; USTTB, DER de Santé publique.

Conflit d'intérêt :

Les auteurs ne déclarent aucun conflit d'intérêt.

Liste des tableaux et figures :

Tableau I : Caractéristiques sociodémographiques des enquêtes en Commune VI de Bamako de 2014 à 2018

\begin{tabular}{|c|c|c|c|}
\hline Caractéristiques & Positif & Négatif & $p$ \\
\hline \multicolumn{4}{|l|}{ Age } \\
\hline Moyenne & 4,98 & 5,50 & 0,55 \\
\hline Médiane & 3,00 & 5,00 & \\
\hline Mode & 1,00 & 5,00 & \\
\hline Etendue & $0,16-31,00$ & $0,16-35,00$ & \\
\hline Ecart Type & 6,93 & 4,80 & \\
\hline \multicolumn{4}{|l|}{ Sexe } \\
\hline Masculin & $19(18,10)$ & $86(81,90)$ & 0,99 \\
\hline Féminin & $20(18,52)$ & $88(81,48)$ & \\
\hline \multicolumn{4}{|l|}{ Profession } \\
\hline Aide-ménagère & $0(00)$ & $3(100)$ & 0.31 \\
\hline Elève & $2(9.52)$ & $19(90.48)$ & \\
\hline
\end{tabular}


MALI SANTE PUBLIQUE 2020 SOMASAP

\begin{tabular}{cll}
\hline Caractéristiques & Positif & Négatif \\
\hline Enfant & $34(18.99)$ & $145(81.01)$ \\
Ménagère & $0(00)$ & $2(100)$ \\
Nourrisson & $2(28.57)$ & $5(71.43)$ \\
Commerçant & $1(100)$ & $0(00)$ \\
Résidence & & \\
1008 logements & $0(00)$ & $5(100)$ \\
759 logements & $0(00)$ & $1(100)$ \\
Baguinéda & $0(00)$ & $2(100)$ \\
Banakabougou & $2(15.38)$ & $11(84.62)$ \\
Dianéguéla & $2(28.57)$ & $5(71.43)$ \\
Faladié & $3(11.54)$ & $23(88.46)$ \\
Kalabancoro & $0(00)$ & $1(100)$ \\
Magambougou & $0(00)$ & $21(100)$ \\
Niamakoro & $6(22.22)$ & $21(77.78)$ \\
Sénou & $12(42.86)$ & $16(57.14)$ \\
Missabougou & $4(25)$ & $12(75)$ \\
Sogonico & $3(16.67)$ & $15(83.33)$ \\
Sokorodji & $3(23.08)$ & $10(76.92)$ \\
Yirimadio & $4(11.76)$ & $30(88.24)$ \\
Centre de notification & & \\
ANIASCO & $0(00)$ & $1(100)$ \\
ASACOBAFA & $0(00)$ & $17(100)$ \\
ASACOYIR & $1(5.74)$ & $18(94.26)$ \\
ASACOMA & $2(33.33)$ & $4(67.67)$ \\
ASACOSO & $2(50)$ & $2(50)$ \\
ASACOCY & $0(00)$ & $4(100)$ \\
ASACOMIS & $2(16.67)$ & $10(83.33)$ \\
ASACOSE & $11(61.11)$ & $7(38.89)$ \\
INRSP & $3(1.72)$ & $1(2.56)$ \\
CSRéf C 6 & $17(15.74)$ & $91(84.26)$ \\
CSRéf C 4 & $1(100)$ & $0(00)$ \\
CSRéf C 5 & $1(50)$ & $1(50)$ \\
CSRéf C 3 & $1(100)$ & $0(00)$ \\
Dembanyuma & $1(16.67)$ & $5(83.33)$ \\
ASACOFA & $1(10)$ & $9(90)$ \\
\hline & & \\
\hline & & \\
\hline
\end{tabular}

Tableau II : Durée moyenne entre le début et la notification de la maladie chez les enquêtes en Commune VI de Bamako de 2014 à 2018

\begin{tabular}{cll}
\hline Durée & Positif & Négatif \\
\hline Moyenne & 2.62 & 2.93 \\
Médiane & 3 & 2 \\
Mode & 1 & 2 \\
Etendue & $0-7$ & $0-30$ \\
Ecart Type & 1.69 & 3.57 \\
\hline
\end{tabular}




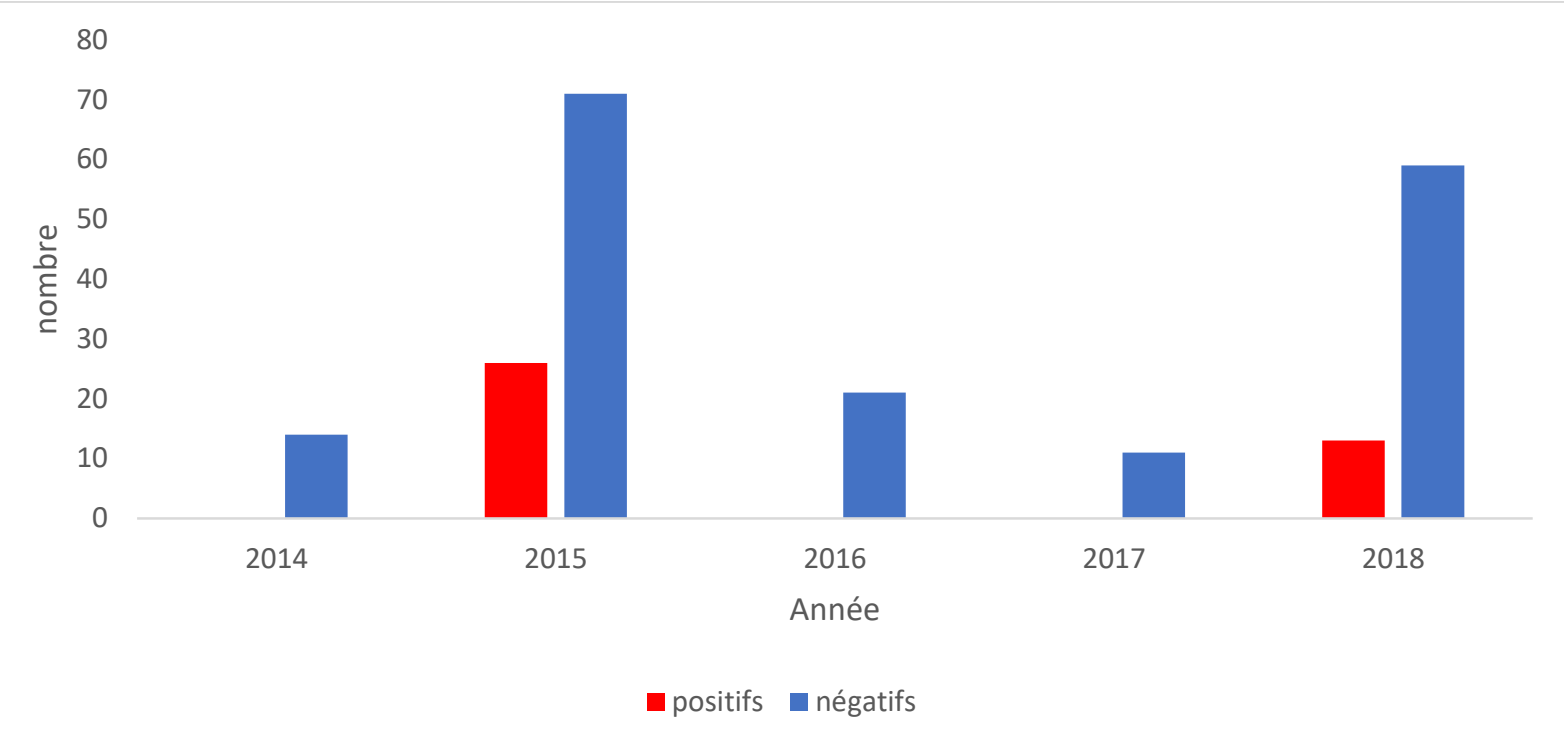

Figure 1 : Répartition des cas positifs et négatifs de 2014 à 2018 en commune VI

Figure 2 : Répartition des cas selon le statut vaccinal

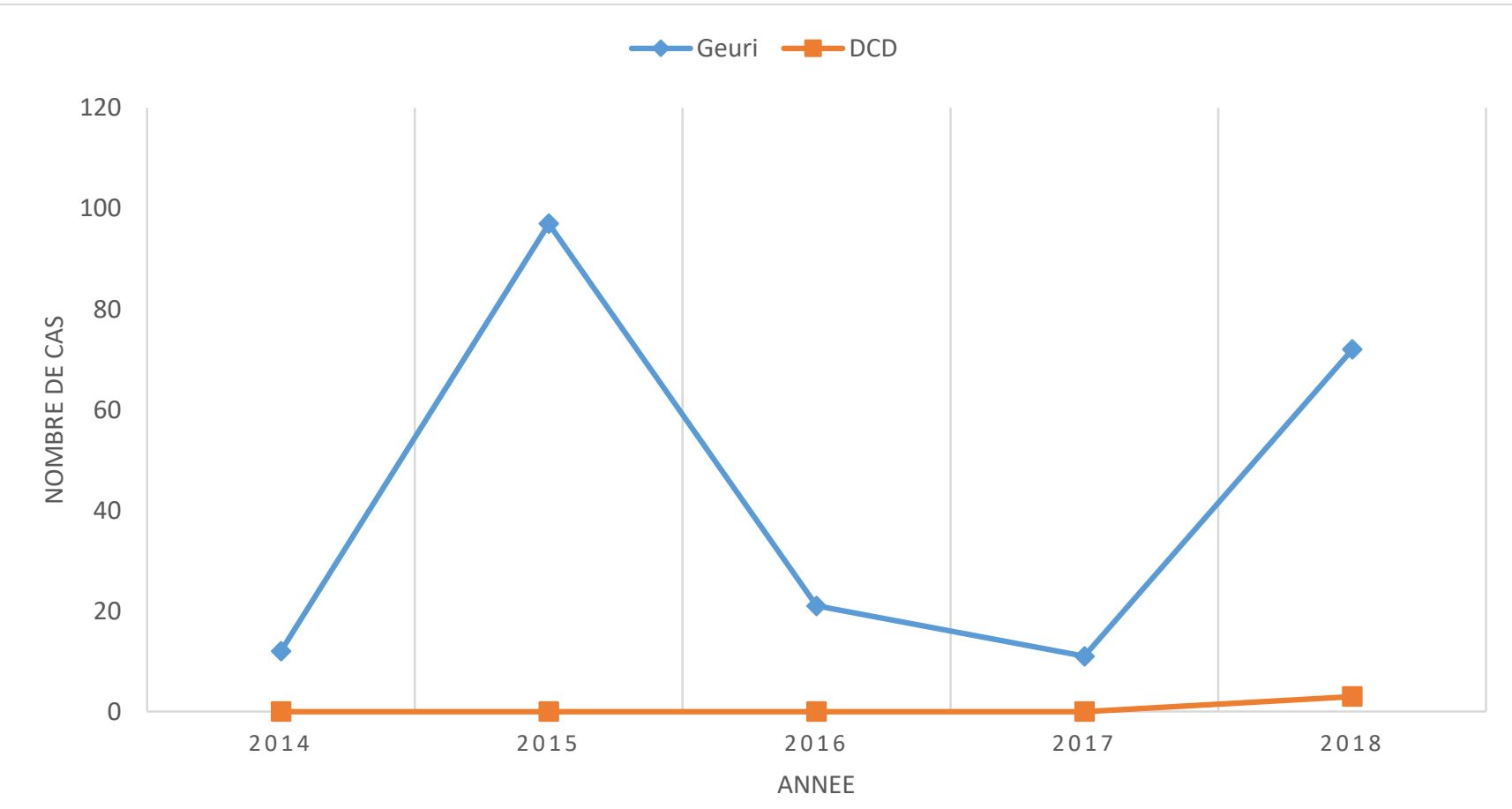

Figure 3 : Répartition des cas selon l'évolution de la maladie 